Portland State University

PDXScholar

Electrical and Computer Engineering Faculty

Publications and Presentations

$11-1-1987$

\title{
Pulse-train instabilities in a mode-locked argon laser: experimental studies
}

Duncan Leo MacFarlane

Lee W. Casperson

Portland State University

Follow this and additional works at: https://pdxscholar.library.pdx.edu/ece_fac

Part of the Electrical and Computer Engineering Commons

Let us know how access to this document benefits you.

\section{Citation Details}

D. L. MacFarlane and Lee W. Casperson, "Pulse-train instabilities in a mode-locked argon laser: experimental studies," J. Opt. Soc. Am. B 4, 1777-1780 (1987).

This Article is brought to you for free and open access. It has been accepted for inclusion in Electrical and Computer Engineering Faculty Publications and Presentations by an authorized administrator of PDXScholar. Please contact us if we can make this document more accessible: pdxscholar@pdx.edu. 


\title{
Pulse-train instabilities in a mode-locked argon laser: experimental studies
}

\author{
D. L. MacFarlane and Lee W. Casperson \\ Department of Electrical Engineering, Portland State University, Portland, Oregon 97207
}

Received March 13, 1987; accepted July 14, 1987

\begin{abstract}
The output train of pulses from an acousto-optically mode-locked argon-ion laser was studied as a function of the loss modulation frequency for small detunings from the optimum $c / 2 L$ modulation. A shift away from the optimum frequency by as little as 1 part in $10^{4}$ was found to produce several strong lower-frequency components that amplitude modulate the mode-locked pulse train. Notably, the strongest subharmonic is a frequency near $f_{\text {opt }} / 4$. Further detuning results in an irregular, slow (of the order of $10^{5} \mathrm{~Hz}$ ) amplitude envelope on the pulse train. The homodyne spectrum of this phase of operation represents a progressive filling-in behavior reminiscent of deterministically chaotic systems.
\end{abstract}

\section{INTRODUCTION}

Since the first reports of active mode locking in lasers more than two decades ago, ${ }^{1}$ this technique of producing a perfectly repetitive train of ultrashort pulses has been developed into a routine method of operation for many commercial laser systems. Since many mode-locking applications demand pulse-to-pulse consistency, much progress has been made in avoiding unpredictable and irregular mode-locked laser outputs. Meanwhile, work in the theoretical arena has focused on similar goals, and several general models now exist that are valid and useful for optimizing active modelocked laser operation. ${ }^{2-5}$ In spite of these efforts, there remain several types of mode-locked lasers in which the observed pulsations are not exactly periodic and have not yet been rigorously interpreted in terms of a theoretical model. In the case of self-locked xenon lasers, for example, the slow chaotic envelope modulation that is sometimes observed $^{6}$ has not yet been modeled. Irregular pulse trains and subharmonic phenomena have also been seen in extended cavity semiconductor lasers ${ }^{7}$ and in actively mode-locked lasers such as synchronously pumped dye lasers ${ }^{8-10}$ when the modulation frequency is not properly matched to the pulserepetition frequency. These effects in driven laser oscillators are analogous to the chaotic behavior of driven electronic oscillators first studied by van der Pol and van der Mark. ${ }^{11}$ Our understanding of the synchronous gain or loss modulation of a laser cannot be considered complete until the structure of such imperfect mode locking is understood.

With the above in mind, we have carried out a systematic experimental study of an imperfectly mode-locked laser. In particular, we report the effect of small detunings of the modulation frequency on the output pulse train of an acousto-optically mode-locked argon laser. The quantities studied include the peak and average powers, the energy per pulse, and the temporal pulse shape. Additionally, the character of the pulse train was monitored using a real-time oscilloscope and a homodyne spectrum analyzer. From these data, the different phases of operation can be classified with respect to the modulation frequency. We find that decreases in the modulation frequency of as little as 1 part in
$10^{4}$ produce strong lower-frequency components that modulate the amplitude of the train of ultrashort pulses. The strongest of these components has a frequency of about 20.5 $\mathrm{MHz}$, which is close to the period-four ${ }^{12}$ frequency of $c / 2 L$, which is approximately $81 \mathrm{MHz}$. As the modulation frequency is further decreased, a slow amplitude envelope of several hundred kilohertz develops on the pulse train. At first, this amplitude modulation is fairly regular in frequency, but, on further detuning, the modulation depth approaches unity and all frequencies less than about $600 \mathrm{kHz}$ are represented. This filling in of the homodyne power spectrum is also found on the immediate high side of the optimum modulation frequency and is characteristic of other deterministically chaotic systems. ${ }^{12,13}$ Notable too is the increase in average power when a new frequency component appears. Similar increases in output power at phase transitions were noted in previous studies of laser instabilities. ${ }^{14}$

\section{EXPERIMENTS}

The experiments were performed using a commercially available, Spectra-Physics 2020 argon-ion laser with a 186$\mathrm{cm}$-long stable resonator. The laser was operated in its $\mathrm{TEM}_{00}$ transverse mode. A fused-silica acousto-optic modulator positioned adjacent to the high-reflectivity mirror was driven at approximately $40 \mathrm{MHz}$ to mode lock the laser. This prism also served to limit laser oscillation to the 514-nm line. A typical average output power for this system is 400 $\mathrm{mW}$, as measured with a Scientech 362 calorimeter.

The beam was sampled using two fast Si P-I-N diodes (Antronics S-2), operating photoconductively with rise times shorter than 35 psec. One of the signals was fed to a Tektronix 7L12 spectrum analyzer in a 7613 mainframe. This setup allowed constant monitoring of the homodyne spectrum as a function of the modulation frequency. The second photodiode was used to study the temporal output. The pulse train was monitored using a Tektronix 7834 storage scope, which was essential in viewing some of the irregular (nontriggerable) signals. A composite pulse was also constructed using a Tektronix 7S11 sampling plug-in with 
an S-4 head. This system permitted an accurate representation of the $<100$-psec mode-locked pulses.

The acousto-optic prism was driven by a standard Spectra-Physics $452 \mathrm{~A}$ mode-locker driver with a 453 stabilizing unit. Our control parameter was the modulation frequency that was controllable to a resolution of $<200 \mathrm{~Hz}$, or about 2 parts in $10^{5}$. Since a standing acoustic wave builds in the prism, this frequency is exactly half of the modulation frequency of the laser. The mode locker was kept in thermal equilibrium at all times in this experiment. ${ }^{15}$

Since this experiment studies the frequency content of the acousto-optic-modulator/argon-laser system, it was important to determine directly the frequencies present in the acousto-optic modulator alone and thereby to ensure that the new frequency content did not arise solely from some anomaly in the acousto-optic modulator. This characterization was done in several ways. Most directly, a helium-neon alignment laser was used to probe the oscillating prism over the driving range of the argon-laser experiments. The helium-neon beam was directed into the prism, off the high- reflectivity mirror, and back out through the prism. This output was directed into one of the fast photodiodes, and the induced modulation was studied using a real-time oscilloscope and a spectrum analyzer. Aside from the fundamental $81-\mathrm{MHz}$ modulation, there were no frequency components commensurate with the subharmonic frequencies present in the argon-laser experiment. In this manner, the modulation depth impressed on the laser beam was measured to be about $60 \%$ at a rf power of $0.9 \mathrm{~W}$. Additionally, no frequencies of interest in the argon system were found on the driving voltage of the acousto-optic crystal when that was monitored, and no high-frequency components were observed on the argon discharge voltage.

\section{RESULTS}

Figures 1(a)-1(d) are sampling-scope composites of the mode-locked pulse at various phases of laser operation determined by frequency detuning, which we define as

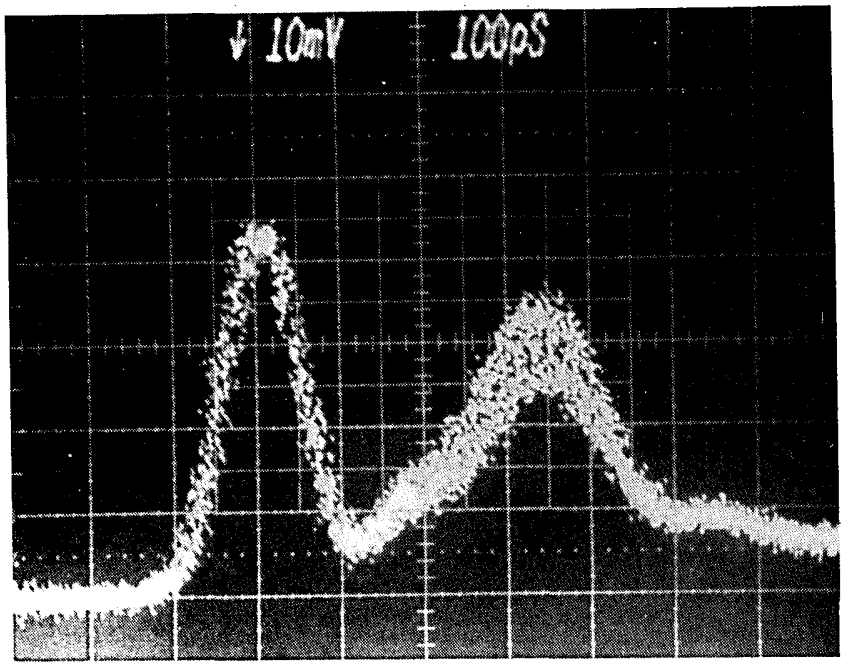

(c)

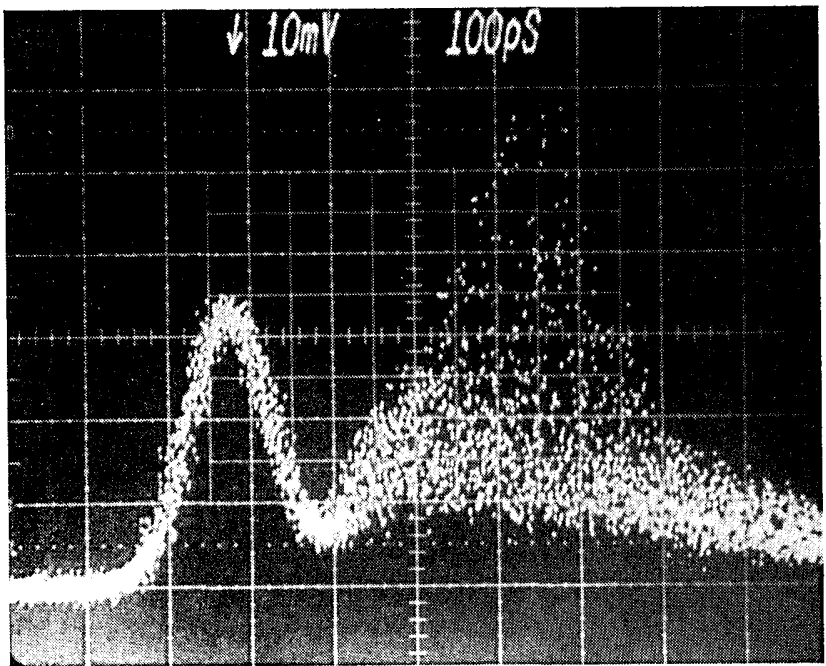

(d)

(b)

Fig. 1. Sampling-scope temporal traces of mode-locked laser operation at various detunings. (a) $\delta=0.0$ (optimum mode-locked operation). (b) $\delta=6.1 \times 10^{-5}$. (c) $\delta=12.2 \times 10^{-5}$. (d) $\delta=15.9 \times 10^{-5}$. Horizontal scale is $100 \mathrm{psec} /$ division in all cases. 


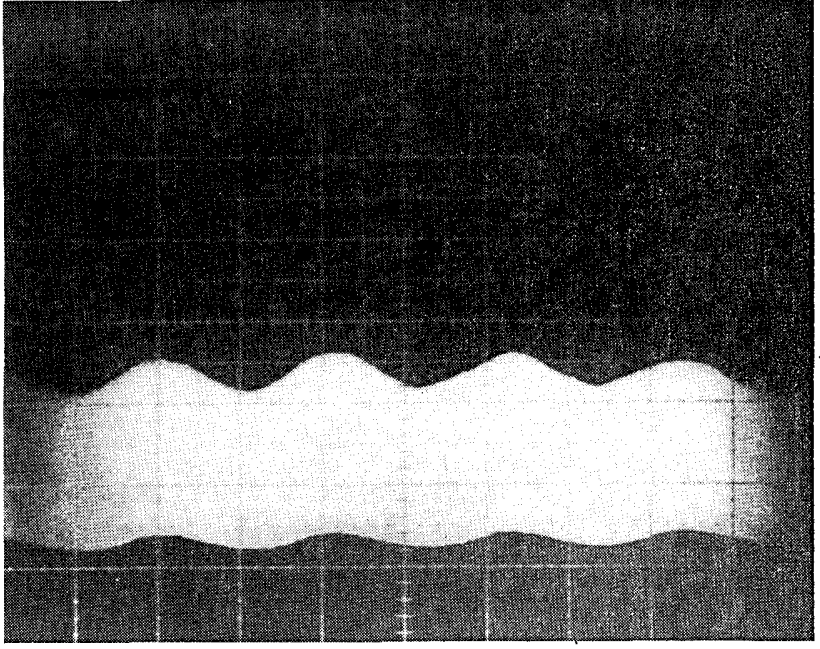

(a)

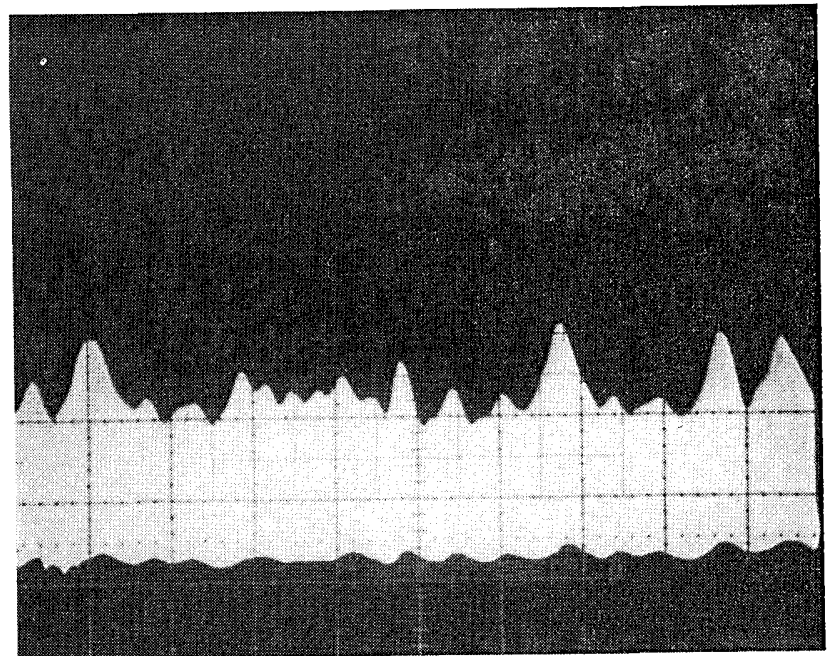

(b)

Fig. 2. Amplitude modulation of mode-locked pulse train. (a) Periodic envelope at $\delta=19.6 \times 10^{-5}(2 \mu \mathrm{sec} /$ division $)$. (b) Chaotic pulse envelope at $\delta=22.0 \times 10^{-5}(5 \mu \mathrm{sec} /$ division $)$.

$$
\delta=\left(f_{\mathrm{opt}}-f\right) / f_{\mathrm{opt}}
$$

Even though these traces represent the superposition of many pulses that at times in this experiment were not identical, much can still be inferred from them. Figure 1(a) represents an optimized ultrashort laser pulse emitted when the acousto-optic modulator is driven at $f_{\text {opt }}$. Figure 1(b) shows the pulse at a modulator frequency that is $2.5 \mathrm{kHz}$ below $f_{\text {opt }}$, a detuning of $\delta=6.1 \times 10^{-5}$. The output now consists of a significant second pulse trailing the original by 450 psec. It is this pulse that is modulated with any further detuning. This $\delta$ is the threshold for new frequency components. The effect of this modulation is shown in Fig. 1(c), taken at $\delta=$ $12.2 \times 10^{-5}$. The spread in the amplitude of the second pulse is interpreted as a fast change in intensity from one pulse to another. The character of this pulse-to-pulse variation can be found from spectrum-analyzer and real-time integrated-pulse measurements. From these, we determine that the second pulse is varying regularly at a frequency of
$20.5 \mathrm{MHz}$. Agreement in the modulation depths calculated from all three types of data corroborates this interpretation.

A second phenomenology occurs at a $\delta$ of $15.9 \times 10^{-5}$. The sampling-scope trace of Fig. 1(d) appears to be just an extreme case of Fig. 1(c); but while there is a strong $20.5-\mathrm{MHz}$ frequency component still present, there is also a slow (100$600-\mathrm{kHz}$ ) modulation of nearly $75 \%$. Moreover, as the detuning is increased, this envelope becomes more irregular, and the initially clean frequency spectrum fills in. Figures 2(a) and 2(b) show the slow envelope at detunings of $19.6 \times$ $10^{-5}$ and $22.0 \times 10^{-5}$, respectively. At a time base of a few microseconds per division, the $81-\mathrm{MHz}$ pulse train is unresolved but present. In Figs. 3(a) and 3(b) are the laser spectra about $81 \mathrm{MHz}$ corresponding to the detunings of Fig. 2. Of note in Fig. 3(b) is the lifted baseline, indicating that all frequencies less than approximately $400 \mathrm{kHz}$ are present.

Interestingly, the phenomenology described above is not mirrored when the modulation frequency is greater than the
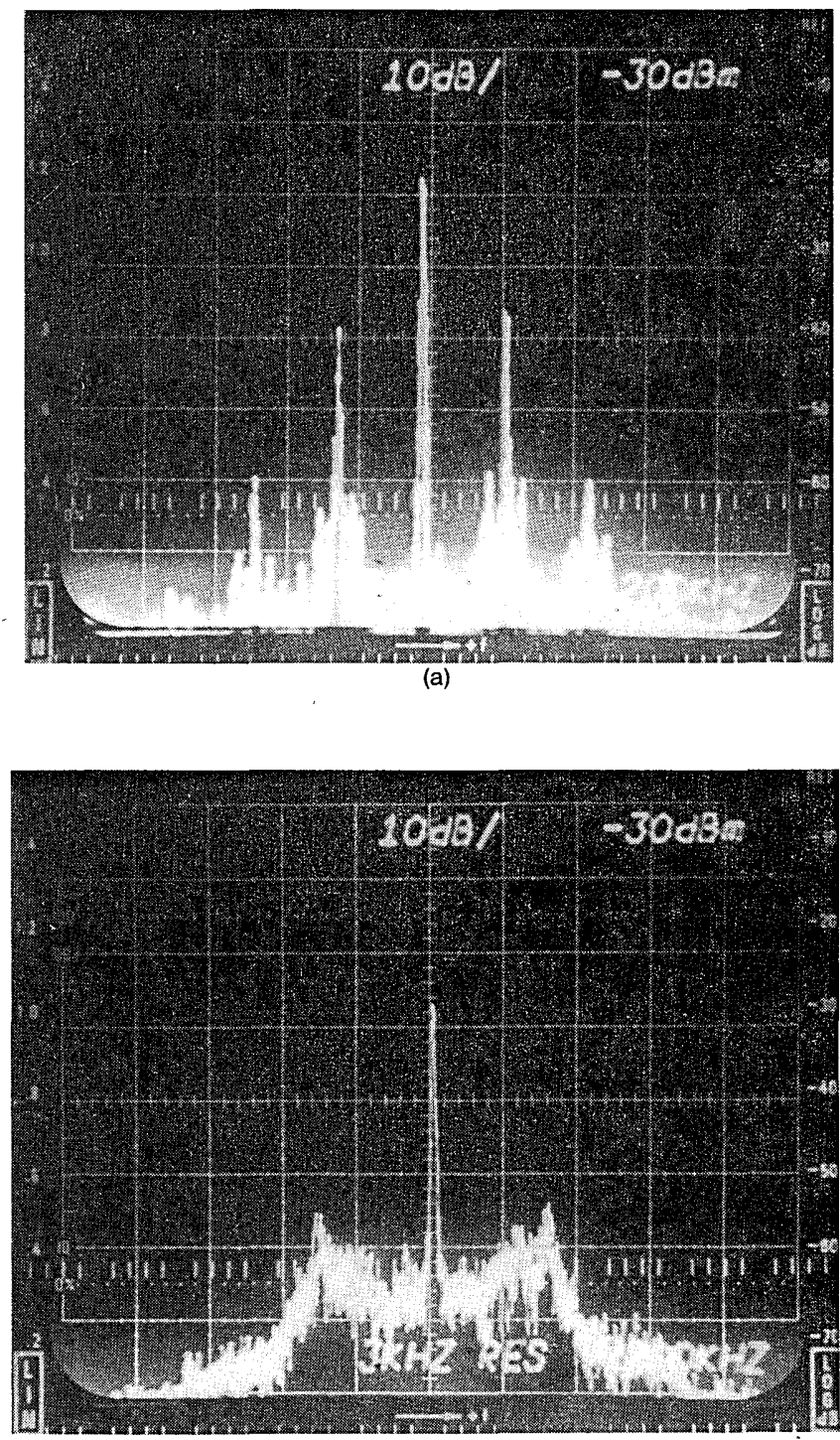

(b)

Fig. 3. Homodyne spectra of slow envelope about $81 \mathrm{MHz}(200$ $\mathrm{kHz}$ /division, $3-\mathrm{kHz}$ resolution, and $10 \mathrm{~dB} /$ division): (a) clean sidebands at $\delta=19.6 \times 10^{-5}$, (b) filled-in modulation at $\delta=22.0 \times$ $10^{-5}$. 


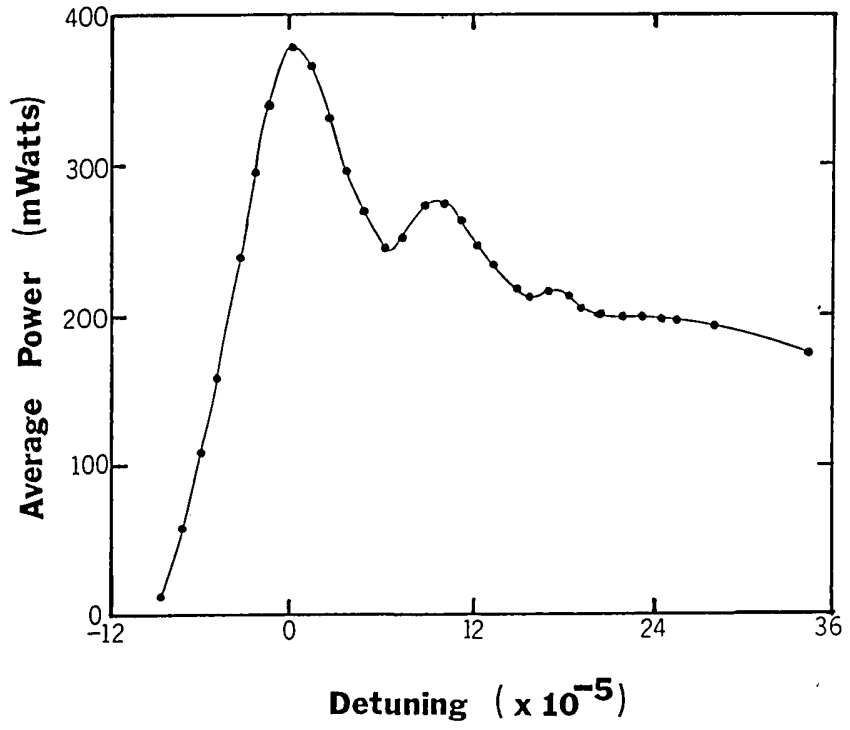

Fig. 4. Average power versus detuning $(\delta)$ showing increases in power at the critical transition detunings, $\delta=6.1 \times 10^{-5}$ and $\delta=16.0$ $\times 10^{-5}$.

optimum. Instead we see a rapid degradation in lasing output as the modulation frequency increases. Additionally, the ultrashort pulses become chaotic almost immediately. There is no second pulse development; however, there is the same erratic, slow envelope shown in Figs. 2(b) and 3(b).

The transitions between the three phases of operation described here can also be illustrated by studying the average power as a function of detuning. Such a plot is shown in Fig. 4. Of interest are the increases in power at $\delta=6.1 \times$ $10^{-5}$, the transition point for the $20.5-\mathrm{MHz}$ modulation, and at $\delta=16 \times 10^{-5}$, the onset of the slow modulation at several hundred kilohertz.

\section{DISCUSSION}

We have studied the output of an imperfectly mode-locked argon laser in the regime of small detunings from the optimum loss modulation frequency. We observed a change in the temporal shape of the pulse with the onset of an echo pulse that lags the original pulse by 450 psec. With further detuning, the energy in this second pulse is modulated, first at a frequency approximately equal to $f_{\text {opt }} / 4$ and later at much slower frequencies $\left(10^{5} \mathrm{~Hz}\right)$. This slow modulation is initially a fairly clean amplitude modulation; however, with further detuning, the envelope becomes chaotic as more sideband frequencies appear.

A heuristic argument may be formulated to explain at least the $f / 4$ bifurcation. This picture treats the laser as a loss-driven oscillator with a nonlinearity due to a saturating gain. In inhomogeneous lasers, the gain saturates as $(1+$ $s I)^{-0.5}$. This form is similar to the nonlinearity found in varactor diodes, and a rich array of subharmonic bifurcation schemes were observed in RLC/varactor diode circuits. ${ }^{16}$ Preliminary theoretical work to explain mode-locking irregularities as a consequence of nonlinear laser dynamics is now under way.

\section{ACKNOWLEDGMENT}

This research was supported in part by the National Science Foundation and by Tektronix, Inc.

\section{REFERENCES}

1. L. E. Hargrove, R. L. Fork, and M. A. Pollack, "Locking of HeNe laser modes induced by synchronous intracavity modulation," Appl. Phys. Lett. 5, 4-5 (1964).

2. O. P. McDuff and S. E. Harris, "Nonlinear theory of the internally loss-modulated laser," IEEE J. Quantum Electron. QE-3, 101-111 (1967).

3. S. E. Harris and O. P. McDuff, "Theory of FM laser oscillation," IEEE J. Quantum Electron. QE-1, 245-262 (1965).

4. D. J. Kuizenga and A. E. Siegman, "FM and AM mode-locking of the homogeneous laser-Part 1: theory," IEEE J. Quantum Electron. QE-6, 694-708 (1970).

5. A. E. Siegman and D. J. Kuizenga, " Modulator frequency detuning effects in the FM mode-locked laser," IEEE J. Quantum Electron. QE-6, 803-808 (1979).

6. L. W. Casperson and A. Yariv, "The time behavior and spectra of relaxation oscillations in a high gain laser," IEEE J. Quantum Electron. QE-8, 69-73 (1972).

7. T. Mukai and K. Otsuka, "Subharmonic oscillations and chaos in a laser diode coupled to an external cavity," in Optical Instabilities, R. W. Boyd, M. G. Raymer, and L. M. Narducci, eds. (Cambridge U. Press, Cambridge, 1986), pp. 297-299.

8. A. Scavennec, "Mismatch effects in synchronous pumping of the continuously operated mode-locked dye laser," Opt. Commun. 17, 14-17 (1976).

9. J.P. Zheng, U. Sen, D. M. Benenson, and H. S. Kwok, "Observation of periodicity in a synchronously pumped dye laser," Opt. Lett. 11, 632-634 (1986).

10. J. Kluge, D. Weichert, and D. von Der Linde, "Fluctuations in synchronously mode-locked dye lasers," Opt. Commun. 51, 271277 (1984).

11. B. van der Pol and J. van der Mark, "Frequency demultiplication," Nature 120, 363-364 (1927).

12. M. J. Feigenbaum, "Universal behavior in nonlinear systems," Los Alamos Sci. 1, 4-27 (1980).

13. C. Sparrow, "The Lorenz model," in Optical Instabilities, R. W. Boyd, M. G. Raymer, and L. M. Narducci, eds. (Cambridge U. Press, Cambridge, 1986), pp. 72-84.

14. L. W. Casperson, "Spontaneous coherent pulsations in ringlaser oscillators," J. Opt. Soc. Am. B 2, 62-72 (1985).

15. L. W. Casperson, L. M. Davis, and J. D. Harvey, "Thermal hysteresis in acoustic resonators," J. Acoust. Soc. Am. 71, 14121416 (1982).

16. P. S. Linsay, "Period doubling and chaotic behavior in a driven anharmonic oscillator," Phys. Rev. Lett. 47, 1349-1352 (1981). 